\title{
Efecto del medio extensor y de las curvas de enfriamiento y congelamiento en la célula espermática en caballos criollos colombianos
}

\author{
Effect of the extender medium and the cooling and freezing curves over the \\ sperm cell in Colombian creole horses
}

\author{
Pérez Osorio, Jair ${ }^{1 *}$ Ph.D; Otero Arroyo, Rafael ${ }^{4}$, Ph.D; Cardona-Alvarez, José ${ }^{3}$ Ph.D; \\ Ospitia, Paula ${ }^{4}$ MV, González, Alejandra ${ }^{4}$ MV, Paredes Cañón, Astri MV.
}

${ }^{1}$ Universidad de La Salle, Facultad de Ciencias Agropecuarias, Bogotá, Colombia. ${ }^{2}$ Universidad de Sucre. Facultad de Ciencias Agropecuarias, Departamento de Zootecnia.

Grupo de Investigación Mejoramiento y Reproducción Animal Sincelejo, Colombia.

${ }^{3}$ Universidad de Córdoba, Facultad de Medicina Veterinaria y Zootecnia, Grupo de Investigación en Medicina de Grandes Animales (MEGA), Montería, Colombia. ${ }^{4}$ Médico Veterinario, Universidad de La Salle, Bogotá - Colombia

\section{KEYWORDS:}

Cryopreservation; dimethylformamide; equine;

glycerol; semen.

\begin{abstract}
The aim was to analyze the parameters of motility, vigor, morphology and integrity of the plasma membrane for each of the cryoprotectans. Each eyaculated was divided into two equal aliquots. One half received dimethylformamide (5\%) and the other half received glycerol $(5 \%)$ as cryoprotectans. The protocol was performed with a cooling curve of $5^{\circ} \mathrm{C}$ for 120 minutes, and exposure to nitrogen vapor for 15 minutes before being submerged in liquid nitrogen. Sperm viability (motility) was assessed by a thermal resistance test, the morphology of cells was evaluated by setting the semen in saline formalin and the integrity of the plasma membrane of the sperm tail by hyposmotic test. In respect to the parameters the post-thawing evaluation of the semen displayed no significant difference $(p>0.05)$ between the two cryoprotectans. The results allow us to conclude that the use of extensor medium INRA 82, modified with sugar associated with skim milk and addition of $2 \%$ egg yolk and moderate cooling curve of $5^{\circ} \mathrm{C}$ for 120 minutes shows a beneficial effect on sperm parameters in post-thaw period independently of the cryoprotectant used in the colombian criollo horses.
\end{abstract}

\section{RESUMEN}

El objetivo fue analizar los parámetros de motilidad, vigor, morfología e integridad de membrana plasmática para cada uno de los crioprotectores. Cada eyaculado se dividió en dos alícuotas iguales al azar con variaciones en la adición del crioprotector (dimetilformamida $5 \%$ o glicerol $5 \%$ ) y se congelo por el protocolo de criopreservación, con una curva de enfriamiento de $5^{\circ} \mathrm{C}$ durante 120 minutos y exposición a vapor de nitrógeno durante 15 minutos antes de ser sumergido al nitrógeno líquido. La viabilidad espermática (motilidad) fue evaluada mediante el test de termorresistencia, la morfología de las células se evalúo fijando el semen en formol salino y la integridad de la membrana plasmática de la cola del espermatozoide mediante el test hiposmótico. Al evaluar el semen pos descongelación no se observó diferencia significativa ( $p>0.05$ ) entre los dos crioprotectores respecto a los parámetros evaluados. Los resultados nos permiten concluir que la utilización del medio extensor INRA 82 modificado con azúcares asociado a leche descremada con adición del $2 \%$ de yema de huevo, y a una curva de enfriamiento moderada de 120 minutos a $5^{\circ} \mathrm{C}$ muestran efectos benéficos en los parámetros espermáticos pos descongelamiento de forma independiente al crioprotector utilizado en los caballos criollos colombianos. 


\section{INTRODUCCIÓN}

La calidad del semen es uno de los factores más importantes en el éxito de programas de biotecnologías reproductivas; dentro de estos programas se encuentra la inseminación artificial con semen congelado, la cual ha generado un gran impacto en la industria equina. La criopreservación del semen permite conservar la viabilidad espermática durante largos períodos de tiempo (Pérez, 2006), este proceso ofrece ventajas tales como el mantenimiento de la diversidad genética en las poblaciones de animales domésticos sin verse afectado por barreras internacionales de transporte, fácil distribución de líneas genéticamente superiores y el tratamiento de la infertilidad iatrogénica, entre otras (WALTERS et ál., 2009).

La investigación sobre protocolos de criopreservación del semen equino ha crecido en las últimas décadas gracias a la aceptación del semen congelado por parte de las grandes asociaciones equinas, sin embargo constantemente se modifican estos protocolos para obtener mejoras de la viabilidad espermática y fertilidad del semen al momento de descongelar (STORNELLI et ál., 2005), lo anterior debido a la alta sensibilidad qué presenta la célula espermática equina durante el proceso de criopreservación (BRINSKO y VARNER, 1992; NEIRA et ál., 2007).

Según PALMA y BRASS (2001), "una variedad de protocolos está disponible para la criopreservación de semen equino, sin embargo no hay estudios comparativos controlados qué indiquen cual es la técnica más eficiente". Por lo anterior se hace necesario invertir en investigaciones qué aporten datos comparativos y controlados acerca de los protocolos de criopreservación más utilizados en el medio equino colombiano, por esto el objetivo de este estudio fue evaluar y comparar el efecto de dos crioprotectores, dimetilformamida y glicerol, en el medio de congelación para semen equino INRA 82 modificado con azúcares, asociados a las curvas de enfriamiento y congelamiento sobre la célula espermática de caballos criollos colombianos.

\section{MATERIALES Y MÉTODOS}

El estudio se realizó en la sabana de Bogotá; municipios de Cajicá, Tabio, Tenjo y El Rosal (CundinamarcaColombia). Se encuentran a una altura promedio de $2600 \mathrm{msnm}$, precipitación media anual de $1.013 \mathrm{~mm}$ y una humedad relativa del $72 \%$ con una temperatura promedio de $13.5^{\circ} \mathrm{C}$ que puede oscilar entre los $-5^{\circ} \mathrm{C}$ y los $26^{\circ} \mathrm{C}$. Geográficamente se encuentra a $4^{\circ} 35^{\prime} \mathrm{LN}$ y $74^{\circ} 04^{\prime}$ LO, con respecto al meridiano de Greenwich.
Se seleccionaron seis sementales criollos colombianos de fertilidad probada, de los cuales a cada uno se le recolectó un eyaculado. Los parámetros de selección dentro de la población fueron: buena condición corporal, edad comprendida entre 4 y 12 años con entrenamiento en la colecta de semen con vagina artificial y buen estado general de salud.

Para la colecta del semen se utilizó una yegua en celo y una vagina artificial (modelo Missouri). Después de la colecta el semen se filtró y fueron evaluados los parámetros seminales de motilidad total y progresiva, vigor, volumen, concentración y $\mathrm{pH}$. El semen qué fue seleccionado para el análisis a una colecta por animal y sólo se utilizaron para la congelación los eyaculados que presentaron motilidad igual o superior al $60 \%$ y una concentración igual o superior a 60 millones de espermatozoides por ml (JASKO et ál., 1994). Estos parámetros fueron analizados en microscopia óptica con un aumento de 400X, utilizando 10 microlitros de semen colocados entre portaobjetos y cubreobjetos atemperados a $37^{\circ} \mathrm{C}$. La motilidad total y progresiva fue determinada por la evaluación del porcentaje de células espermáticas que presenten movimiento circular abierto, en la evaluación de mínimo 6 campos visuales. El vigor o el movimiento de los espermatozoides fue clasificado de cero (ausente) a cinco (máximo). La concentración fue evaluada en la muestra de semen fresco y posteriormente al proceso de la centrifugación, realizando una dilución en una solución de formol salina tamponada en una proporción 1:100 para ser analizada en la cámara de Newbauer, de acuerdo a la fórmula: [ ] = (número de spz contados) x (25) x (100). Para determinar la morfología espermática se evaluaron muestras de semen fresco, colocándose 20 microlitros de semen a la solución de formol salino tamponado (BARTH y OKO, 1989). La evaluación fue realizada en microscopia de contraste de fase y se consideraron mínimo 100 células, de acuerdo al protocolo descrito por NIE y WENZEL (2001).

Posteriormente el semen fue fraccionado en tubos estériles Falcon de $14 \mathrm{ml}$ y suspendido de nuevo en medio extensor (INRA 82 modificado sin crioprotector), y se sometió al proceso de centrifugación a una velocidad de 2500 revoluciones por minuto (rpm), equivalente a 600 gauss en un periodo de tiempo de 12 minutos. Se procedió a retirar el sobrenadante de cada tubo, y se dejó el sedimento para ser suspendido nuevamente en el medio INRA 82 + crioprotector (dimetilformamida $5 \%$ o glicerol $5 \%$ ). Se utilizaron pajillas de $0.5 \mathrm{ml}$ con una concentración de $100 \times 10^{6}$ espermatozoides/ $\mathrm{ml}$, empacadas manualmente y selladas con alcohol polivinil. 
La curva de enfriamiento se realizó durante dos horas hasta alcanzar los $5^{\circ} \mathrm{C}$ (PÉREZ, 2006), intervalo en qué los espermatozoides son sensibles al choque térmico. La curva de congelamiento se realizó exponiendo las pajillas durante 15 minutos a $4 \mathrm{~cm}$ (VIDAMENT et ál., 2001) por encima del nivel del nitrógeno (vapores de nitrógeno) en una caja de icopor, pasado este tiempo se sumergieron en el nitrógeno líquido a una temperatura de $-196^{\circ} \mathrm{C}$ (PALMA, 2001). La curva de descongelación del semen se realizó en dos tiempos; se sumergieron las pajillas en agua a $52^{\circ} \mathrm{C}$ durante 10 segundos e inmediatamente después se pasó a un baño maría a $37^{\circ} \mathrm{C}$ durante 30 segundos (PÉREZ, 2006).

La evaluación del semen incluyó parámetros como motilidad total, motilidad progresiva, vigor, morfología e integridad de la membrana plasmática. Las muestras de semen fueron sometidas a un test de termo resistencia dónde se evalúo la motilidad total, motilidad progresiva y vigor a intervalos de tiempo correspondientes a 30 minutos hasta que el porcentaje de la motilidad disminuyo al 5\% (PÉREZ, 2006). La evaluación de la integridad funcional de la membrana plasmática de la cola de los espermatozoides fue evaluada mediante el test hiposmótico (HOST), el cual consiste en la mezcla de una gota de 100 microlitros de semen en un $\mathrm{ml}$ de solución de sacarosa a $100 \mathrm{mOsm} / \mathrm{L}$, a una temperatura de $37^{\circ} \mathrm{C}$. Posteriormente la muestra fue incubada en baño maría por un periodo de treinta minutos, luego las muestras fueron fijadas en $0.5 \mathrm{ml}$ de solución de formol salino tamponado y se realizó la lectura en microscopia de contraste de fase con un aumento de 1000 veces. Los espermatozoides reactivos al test hiposmótico fueron aquellos que mostraron enrollamiento de cola, lo cual demuestra integridad de la membrana plasmática de la célula. La morfología fue evaluada colocando 30 microlitros de semen en un $\mathrm{ml}$ de formol salino, posterior a esto se observó en el microscopio 10 microlitros de esta solución, contando un total de 100 células y observando cada uno de los defectos morfológicos.

Los datos cuantitativos fueron sometidos a un análisis de verificación de distribución normal mediante la prueba Shapiro-Wills. Fue realizado un análisis de varianza completamente al azar con un grado de significancia del $5 \%(p<0.05)$. Las medias fueron comparadas por la prueba de Tukey. Los datos cualitativos (motilidad total, motilidad progresiva y vigor) fueron analizados mediante la prueba no paramétrica de Kruskal-Wallis y mediante un análisis de regresión lineal simple para establecer la variación de la motilidad espermática con relación al tiempo pos descongelación en función de los dos diferentes crioprotectores evaluados (dimetilformamida, glicerol).

\section{RESULTADOS}

Los parámetros evaluados en el semen fresco se compararon con los datos obtenidos después de la adición del INRA 82 sin crioprotector, también de la adición de los crioprotectores y del semen evaluado pos descongelación. En el análisis las células se vieron afectadas con la adición de los crioprotectores y después de la fase de congelación, este declive de los parámetros espermáticos no mostró diferencias estadísticas ( $p>0.05)$, pero en las figuras 1 y 2 se puede observar la disminución de la motilidad y el vigor espermáticos de acuerdo a cada etapa del proceso.

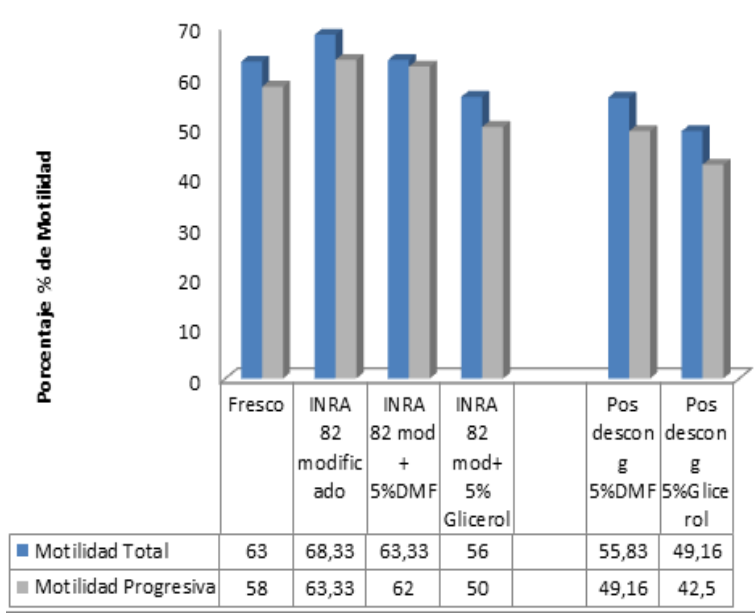

Figura 1. Comparación del efecto de las etapas del proceso de congelación sobre la motilidad de la célula espermática equina.

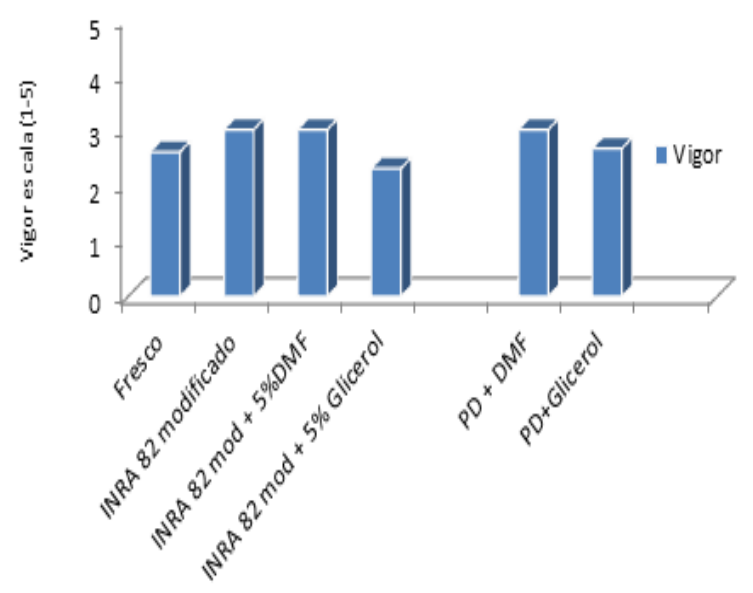

Figura 2. Comparación del efecto de cada proceso de congelación sobre el vigor de la célula espermática equina. 
El análisis del test de termorresistencia en la prueba no paramétrica de Kruskal-Wallis no arrojo diferencia estadísticamente significativa entre los dos crioprotectores ( $p>0.05$ ). La figura 3 representa un análisis de regresión qué establece la variación de la motilidad espermática con relación al tiempo de descongelación en función de los dos crioprotectores, dimetil formamida y glicerol. En relación al vigor espermático no se observó diferencia significativa $(p>0.05)$.
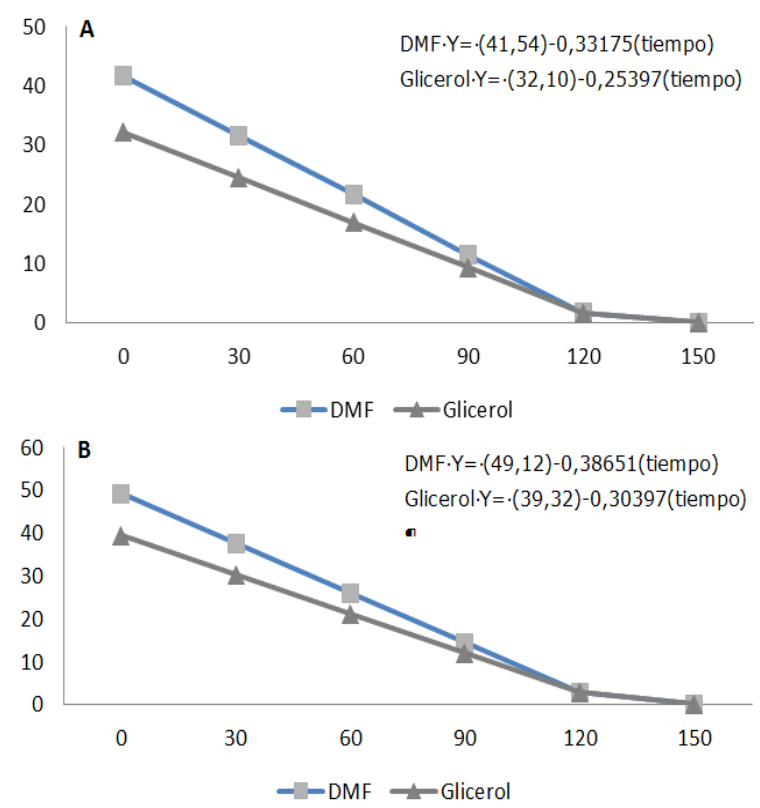

Figura 3. Viabilidad espermática de acuerdo al crioprotector a través del tiempo. La figura $3 \mathrm{~A}$ muestra los porcentajes de motilidad progresiva pos descongelamiento a través del tiempo y la figura 3B muestra el porcentaje de espermatozoides qué presentaron cualquier tipo de movimiento a través del tiempo.

En la evaluación de la morfología se encontró un aumento de las células espermáticas anormales después del proceso de congelación. La figura 4 compara el porcentaje de células normales durante los diferentes tiempos de evaluación.

En los resultados del test hiposmótico para los espermatozoides criopreservados con dimetilformamida el porcentaje de células reactivas con una membrana plasmática íntegra y funcional al minuto cero (0) de ser evaluados fueron en media de $44.16 \%$ variando de $37 \%$ a $54 \%$ y al minuto 30 la media fue de $45.16 \%$ variando de $23 \%$ a $59 \%$. Los espermatozoides criopreservados con glicerol al ser evaluados en el minuto cero (0) tuvieron una media de $48.16 \%$ variando de $34 \%$ a $64 \%$ y al minuto 30 la media fue de $41.5 \%$ variando de $24 \%$ a $53 \%$, dónde no se observó diferencia significativa entre estos dos tratamientos ( $p>0.05$ ). La figura 5 muestra un incremento de células reactivas al HOST del glicerol respecto a la dimetilolformamida en el primer tiempo de evaluación y una caída dramática de células reactivas en la segunda evaluación al minuto 30 , siendo la dimetilformamida superior en esta última evaluación.

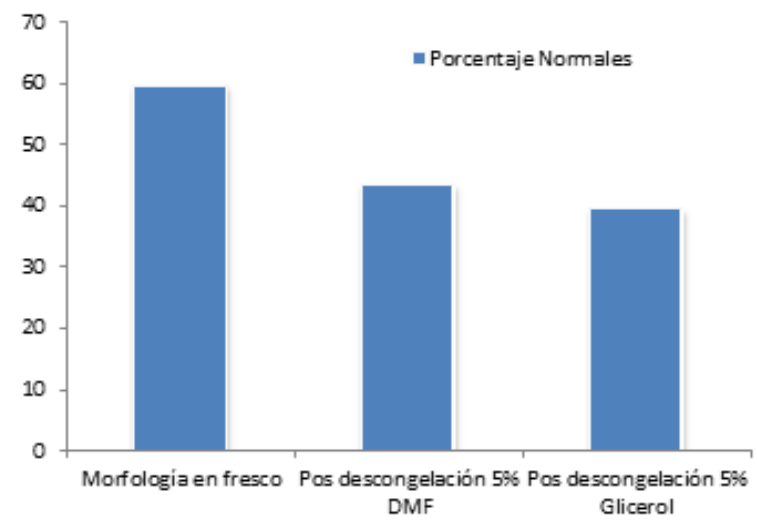

Figura 4. Comparación de la morfología pre y pos congelación.

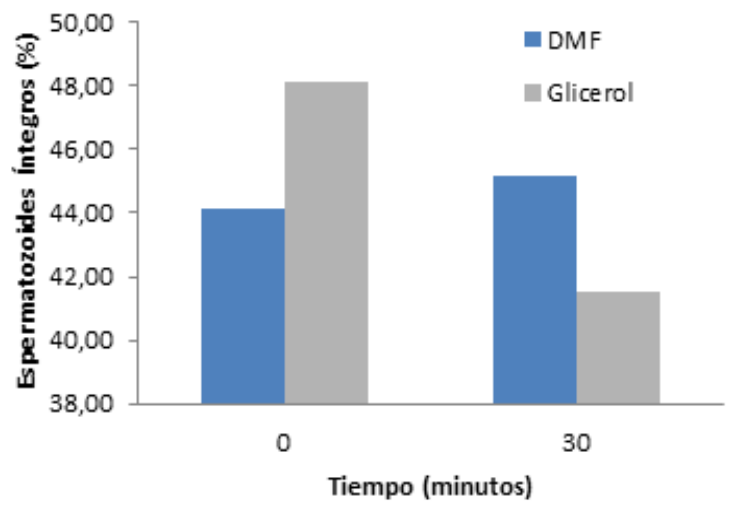

Figura 5. Porcentaje de células reactivas al test hiposmótico.

\section{DISCUSIÓN}

Este estudio se basa en la comparación de dos crioprotectores permeantes como la dimetilformamida y el glicerol. Estudios anteriores han comparado el efecto de estos dos crioprotectores sobre la célula espermática equina (MEDEIROS, 2002) (VIDAMENT, 2002) donde se han evaluado las diferentes concentraciones del agente crioprotector en el medio extensor. GÓMEZ et ál. (2002) demostró qué mayores concentraciones de dimetilformamida como $5 \%$ resultó en un aumento de la motilidad pos descongelación, ALVARENGAet ál. (2003) afirma qué esto se refleja particularmente en caballos qué son considerados malos para la congelación. En este estudio se utilizó la dimetilformamida y el glicerol al $5 \%$ ya que a pesar de no ser reportado, las características seminales del caballo criollo colombiano son consideradas inferiores a las de otras razas, tanto en semen fresco como en el semen congelado (MESA y HENAO, 2012). 
La principal fuente de energía disponible para los espermatozoides se encuentra en los azucares que se adicionan en el medio extensor para la congelación. En el estudio se modificó la composición del medio INRA 82, sustituyendo la rafinosa por dos azúcares, sucrosa y fructosa. Los azúcares monosacáridos como la glucosa y la fructosa son la mayor fuente de energía para los espermatozoides ya que la célula tienen la capacidad de metabolizar estas moléculas rápidamente (ARIFIANTINI, 2010), por esta característica se generó así un medio extensor con una fuente energética enriquecida. Además WOLDERS et al. (1997) demostró qué la sucrosa protege del daño celular por rápido enfriamiento, ya que al ser de un alto peso molecular sirve como crioprotector extracelular. Por estas propiedades osmóticas y crioprotectoras, los azúcares han sido comúnmente usados como crioprotectores no penetrantes combinándolos con crioprotectores penetrantes (ARIFIANTINI, 2010). La adición de estos azúcares al medio extensor fue benéfica sobre los parámetros espermáticos al generar un medio con una fuente energética de fácil acceso para la célula espermática y protección externa mediante una molécula no penetrante que protegió la membrana plasmática durante el proceso de congelación.

En la evaluación de los parámetros de motilidad o viabilidad espermática pos descongelación el uso del glicerol como crioprotector es controversial. Investigaciones anteriores reportan qué su uso puede ser un factor involucrado en bajas tasas de movilidad y vigor espermático (ALVARENGA et ál., 2005). Los resultados obtenidos muestran que la motilidad espermática con dimetilformamida se mantuvo ligeramente más alta durante la evaluación en relación al glicerol, pero para ambos tratamientos la motilidad decayó en el mismo intervalo de tiempo pos descongelación, sin observarse diferencias significativas, lo cual coincide con estudios semejantes donde no encuentran diferencia asociadas al daño del glicerol sobre la membrana espermática cuando se asocia a un medio extensor enriquecido (MORILLO, 2013).

En la evaluación de la morfología celular se observó un aumento de los defectos morfo estructurales en los espermatozoides congelados con glicerol. Las anormalidades más observadas fueron cabezas piriformes, pieza intermediaria y tracto terminal enrollado. Estos cambios pueden darse por la toxicidad del glicerol sobre el espermatozoide, la cual resulta por la desnaturalización de las proteínas, alteraciones de las interacciones de la actina y la inducción de ampollas de membrana libres de proteínas (ALVARENGA et ál., 2005). HAMMERSTEDT (1992) reporta otros efectos celulares causados por el glicerol, los cuales incluyen cambios en eventos citoplasmáticos, dados por la viscosidad del glicerol intracelular, alteración de la polimerización de la tubulina, alteración de la asociación de microtúbulos, efectos en el balance bioenergético y alteración directa sobre la membrana de plasma y glicocalix (ALVARENGA et ál., 2005). Estas alteraciones pueden afectar los parámetros de motilidad y vigor espermáticos, pero cuando KEITH (1998) comparo diferentes amidas como crioprotectores para semen equino, observo que entre las amidas metilformamida y dimetil formamida resulto un más alto porcentaje de la motilidad, y sin embargo la preservación del semen fue similar al glicerol. Por lo cual a pesar de la alta toxicidad reportada para el glicerol, este crioprotector puede generar parámetros de motilidad, vigor y morfología más bajos pero cercanos a los alcanzados por la dimetil formamida.

El comportamiento de las células espermátias en el test hiposmótico puede deberse a la naturaleza del glicerol, ya que al ser una solución isosmótica de solutos polares puede provocar el hinchamiento celular debido a su capacidad de arrastrar agua cuando atraviesan la membrana celular alterando así el equilibrio de las presiones osmóticas internas y externas (HAMMERSTEDT et ál., 1990; RUBIO, 2009), lo cual puede generar un mayor número de células hinchadas, pero esta alteración de equilibrio al transcurrir el tiempo puede afectar mediante un shock osmótico la integridad de la célula generando así un mayor número de espermatozoides dañados qué no son reactivos a la prueba hiposmótica.

Los crioprotectores incorporados a los diluyentes producen un estrés transitorio pero importante sobre la membrana plasmática de los espermatozoides, la magnitud de este hecho está íntimamente relacionada con la capacidad penetrante de los crioprotectores (GAO, 1992; STORNELLI et ál., 2005). Ya qué las amidas tienen un peso molecular bajo en comparación con el glicerol (ALVARENGA et ál., 2005), tienen una mayor facilidad para atravesar la membrana espermática, lo cual resulta en un tiempo de equilibrio más rápido entre la región intra y extra celular generando, en teoría, un daño potencial más bajo de la célula a través del shock osmótico (PÉREZ, 2008). Pero a pesar qué el glicerol produce este estrés osmótico, se ha observado qué esa hiperosmolaridad posee un efecto estimulador de la reacción acrosómica. (STORNELLI et ál., 2005), lo cual favorece la fertilización permitiendo qué el espermatozoide penetre en la zona pelúcida y entre en el óvulo. Tal vez por este hecho se ha reportado qué el glicerol presenta bajas tasas de motilidad pos descongelación frente a la dimetil formamida, pero en su capacidad fecundante in vivo presenta buenas tasas de preñez, y por esta razón sigue siendo utilizado en el medio equino. 
La estabilización de la membrana durante el proceso de enfriamiento es también un factor importante en el éxito de la criopreservación. VARNER et ál. (1988) observó que las tasas de enfriamiento pueden afectar la capacidad de retención de la motilidad. El uso de tiempos de equilibrios largos como 145 minutos mejora la respuesta del semen equino al proceso de criopreservación, evaluado para la motilidad progresiva y total, para vigor espermático, para las características morfológicas y para las pruebas de termorresistencia y HOST (FÜRST, 2006). La curva qué se utilizó fue de 120 minutos, por lo cual los resultados de motilidad, vigor y HOST entre los dos crioprotectores utilizados no difirió, ya qué PÉREZ (2006) afirma qué una estabilización de membrana induce a una mejor motilidad progresiva y una mayor reactividad de la célula frente al HOST independientemente de los crioprotectores utilizados. Además ha sido demostrado qué el rápido enfriamiento en una tasa moderada antes del congelamiento en medios extensores qué contengan leche descremada y $2 \%$ de yema de huevo resulta en un incremento de la motilidad total y progresiva (PÉREZ, 2006). Por lo cual la asociación de los azúcares al medio INRA 82 con una curva de enfriamiento moderada disminuyó los efectos tóxicos del glicerol haciendo qué sus parámetros fueran muy cercanos a los alcanzados por la dimetilformamida.

En conclusión la utilización del medio INRA 82 modificado con azúcares que mejoran la fuente energética y la crioprotección extracelular asociado a leche descremada con $2 \%$ de yema de huevo y a una curva de enfriamiento moderada de 120 minutos a $5^{\circ} \mathrm{C}$ muestran efectos benéficos en los parámetros espermáticos en el periodo pos descongelamiento de forma independiente al crioprotector utilizado en los caballos criollos colombianos.

\section{REFERENCIAS}

ALVARENGA, M.; PAPA, F.; LEÃO, K.; LANDIM, F.; MEDEIROS, A.; GOMES, G. 2003. The use of alternative cryoprotectans for freezing stallion semen. En: Proceedings of a workshop on transporting gametes and embryos. Havemeyer Foundation. USA.

ALVARENGA, M.; PAPA, F.; LANDIM, F.; MEDEIROS, A. 2005. Amides as cryoprotetans for freezing stallion semen: A review. Anim Reprod Sci 89:105-113.

ARIFIANTINI, R.; PURWANTARA, B.; YUSUF, T.; SAJUTHI, D. 2010. Effect of different cryoprotective agents on skim milk and dimitropoulus extender for stallion semen cryopreservation. J Indonesian Trop Anim Agric 35(1): 68-74.

BARTH, A.; OKO, R. 1989. Abnormal morphology of bovine spermatozoa. Ames: lowa University Press.

BRASS K. 2001. Inseminación artificial en la especie equina. Biotecnología de la reproducción. INTA. Argentina.

BRINSKO, S.; VARNER, D. 1992. Artificial Insemination and Preservation of Semen. Stallion Management. Vet Clinics of North America: Equine Practice 8: 205 - 218.

FÜRST, R. 2006. Efeito de diferentes tempos de equilíbrio, taxas de congelamento e concentrações espermáticas na fertilidade do sêmen equino. Universidade Federal de Viçosa. Teses de doutorado.

GAO, D.; MAZUR, T.; KLEINHANS, F.; WATSON, P.; NOILES, E.; CRITSER, J. 1992. Glycerol permeability of human spermatozoa and its activation energy. Rev Criobiology 29: 657-667.

GOMES, G.; JACOB, J.; MEDEIROS, A.; PAPA, F.; ALVARENGA M. 2002. Improvement of stallion spermatozoa preservation with alternative cryoprotectants for the Mangalarga Marchador breed. Theriogenology 58 (2/4): 277-279.

HAMMERSTEDT, R.; GRAHAM, J.; NOLAN, J. 1990. Criopreservation of mammalian sperm: what we ask them to survive. J. Andrology 11: 73-88.

HAMMERSTEDT, R. 1992. Artificial insemination using extended liquid semen: An old technology of great value to modern industry. En: 41st Annual National Breeders Roundtable. Southeastern Poultry and Egg Association. Atlanta. JASKO, D. 1994. Procedures for cooling and freezing of equine semen. Ars Veterinaria 10: 156-165.

KEITH, S. 1998. Evaluation of new crioprotectants for the preservation of equine semen. Fort Collins: Colorado State University. Tesis de Maestría. 
MEDEIROS, A.; GOMES, G.; CARMO, M.; PAPA, F.; ALVARENGA, M. 2002. Cryopreservation of stallion sperm using different amides. Theriogenology 58: 273-276.

MESA, A.; HENAO, R. 2012. Efecto del colesterol y la dimetilformamida sobre parámetros posdescongelación en espermatozoides de caballos criollos colombianos. Rev. MVZ Córdoba 17(1): 2908-2915.

MORILLO, A. 2013. Evaluación de crioprotectores alternativos, glicerol y antioxidantes en la congelación del eyaculado equino. Universidad de Extremadura. Tesis de doctorado.

NEIRA, J.; RAMÍREZ, G.; LEÓN, S.; MORENO, D. 2007. Efecto de la asociación L-Glutamina-Etilenglicol en la criopreservación de semen equino. Rev Universidad de La Salle 14 (2): 93-105.

NIE, G.; WENZEL, J. 2001. Adaptation of the hypoosmotic test to asses functional integrity of stallion spermatozoal plasma membranes. Theriogenology 55: 1005-1018.

PALMA, G. 2001. Biotecnología de la reproducción. INTA. Argentina.

PEREZ, J. 2006. Efeito da adição fracionada de dimetil formamida e das curvas de congelamento na viabilidade in vitro pós-descongelamento do espermatozóide eqüino. Universidad Federal de Minas de Gerais. Tesis de maestría.

PEREZ, J.; MELLO, F.; JULIANI, G.; LAGARES, M.; LAGO, L.; HENRY, M. 2008. Effect on post-thaw viability of equine sperm using stepwise addition of dimethylformamide and varying cooling and freezing procedures. Anim Reprod 5: 103-109.

STORNELLI, M.; TITTARELLI, C.; SAVIGNONE, C.; STORNELLI, M. 2005. Efecto de los procesos de criopreservación sobre la fertilidad seminal. Analect Vet 25 (2): 28-35.

RUBIO, J.; QUINTERO, A.; GONZÁLEZ, D. 2009. Efecto de la criopreservación sobre la integridad de la membrana plasmática y acrosomal de espermatozoides de toros. Rev. Cient 19: 382-389.

VARNER, D.; BLANCHARD, T.; LOVE, C.; GARCIA, M.; KENNEY, R. 1988. Effects of cooling rate and storage temperature on equine spermatozoa motility parameters. Theriogenology 29: 1043-1054.

VIDAMENT, M.; YVON, J.; COUTY, I.; ARNAUD, G.; NGUEKAM, J.; NOUE, P. 2001. Advances in cryopreservation of stallion semen in modified INRA 82. Anim Reprod Sci 68: 201-218.

VIDAMENT, M.; DAIRE, C.; YVON, J.; DOLIGEZ, P.; BRUNEAU, B.; MAGISTRINI, M. 2002. Motility and fertility of stallion semen frozen with glycerol and/or dimethyl formamide. Theriogenology 58: 249-251.

WALTERS, E.; BENSON, J.; WOODS, E.; CRITSER, J. 2009. The history of sperm cryopreservation. In: Pacey AA, Tomlinson MJ, editors. Sperm banking: theory and practice. Cambridge: Cambridge University Press.

WOELDERS, H.; MATTHIJS, A.; ENGEL, B. 1997. Effects of trehalose and sucrose osmolarity of the freezing medium and cooling rate on viability and intactness of bull spem after freezing and thawing. Criobology 35(2): 99-105. 